\title{
TWO UNRELATED RESULTS INVOLVING BAIRE SPACES
}

\author{
H. E. WHITE, JR.
}

Abstract. Two results are obtained in this paper. The first is a generalization of J. C. Oxtoby's category analogue of the Kolmogoroff zero-one law. The second result: every dense $G_{\delta}$ subset of a quasi-regular $\alpha$-favorable space is $\alpha$-favorable.

1. An extension of Oxtoby's category zero-one law. Suppose $X$ is the product of the family $\left(X_{i}\right)_{i \in I}$ of sets. A subset $A$ of $X$ is called a tail set [2] if, whenever $x=\left(x_{i}\right) \in A, y=\left(y_{i}\right) \in X$, and there is a finite set $F$ such that $x_{i}=y_{i}$ whenever $i \in I \sim F$, then $y \in A$.

In [2], J. C. Oxtoby proved the following category analogue of Kolmogoroff's zero-one law in probability.

1.1 TheOrem. Suppose each $X_{i}$ is a topological space with a countable pseudo-base [2].

1.1.1. If $A$ is a tail set which has the property of Baire in $X$, then either $A$ or $X \sim A$ is of the first category in $X$.

The following definition is convenient.

Definition. A topological space $(X, \mathscr{T})$ is said to have property (P) if, whenever $Y$ is a Baire space for which there is a nonempty $U$ in $\mathscr{T}$ such that $U \times Y$ is a Baire space, then $V \times Y$ is a Baire space for every $V$ in $\mathscr{T}$ for which $V$ is a Baire space.

In this section, we shall, utilizing a slight modification of Oxtoby's proof of 1.1 , prove the following statement.

1.2 THEOREM. If each $X_{i}$ has property (P), then 1.1 .1 holds.

The following statement indicates that many spaces have property $(\mathrm{P})$.

1.3 Proposition. If $(X, \mathscr{T})$ satisfies any of the following conditions, then $X$ has property $(\mathrm{P})$.

(1) If $U \in \mathscr{T}$ and $U$ is a Baire space, then $U \times Y$ is a Baire space whenever $Y$ is a Baire space.

Received by the editors June 13, 1973 and, in revised form, August 9, 1973.

AMS (MOS) subject classifications (1970). Primary 54E99, 54D99.

Key words and phrases. Tail set, zero-one law, weakly $\alpha$-favorable, property of Baire, $\alpha$-favorable, pseudo-complete.

(c) American Mathematical Society 1974 
(1a) $\mathscr{T}$ has a locally countable pseudo-base [2].

(1b) $X$ is weakly $\alpha$-favorable [4].

(1c) $X$ is a Borel set in a compact, Hausdorff space $T$.

(2) If $U, V$ are nonempty open subsets of $X$, then there are nonempty $U^{\prime}, V^{\prime}$ in $\mathscr{T}$ such that $U^{\prime} \subset U, V^{\prime} \subset V$, and $U^{\prime}$ is homeomorphic with $V^{\prime}$.

(2a) $X$ is a homogeneous space.

(3) $X$ is the product of a family of spaces, each of which has property (P).

Proof. It is clear that any space which satisfies (1) has property (P). And, it follows from Theorem 2 of [2] (3(9) of [4]), that if $X$ satisfies (1a) ((1b)), then $X$ satisfies (1).

Suppose (1c) holds and that $U \in \mathscr{T}$ such that $U$ is a Baire space. Then $U$ is a dense Borel subset of $T^{\prime}=\mathrm{cl}_{T} U$. Hence $U$ is $\alpha$-favorable (see 2.4 (1)).

If (2) holds and $Y$ is such that $U \times Y$ is a Baire space for some nonempty $U$ in $\mathscr{T}$, then every nonempty $V$ in $\mathscr{T}$ which is a Baire space contains a nonempty open set $V^{\prime}$ such that $V^{\prime} \times Y$ is a Baire space. Hence $X$ has property (P). And, if $X$ satisfies (2a), clearly $X$ satisfies (2).

The proof of (3) is easy and is omitted.

Proof of 1.2. Suppose $A$ is of the second category in $X$. By the Banach category theorem, there is a nonempty basic open set $U$ such that $U \cap A$ is a Baire space. Suppose that $V$ is an open subset of $X$ which is of the second category in $X$. By the Banach category theorem, there is a nonempty basic open set $W$ contained in $V$ such that $W$ is a Baire space. There are a finite subset $F$ of $I$, open sets $U^{*}, W^{*}$ of $\prod_{i \in F} X_{i}$, and a subset $A^{*}$ of $\prod_{i \in I \sim F} X_{i}$ such that $U=U^{*} \times \prod_{i \in I \sim F} X_{i}, W=W^{*} \times \prod_{i \in I \sim F} X_{i}$, and $A=\prod_{i \in F} X_{i} \times A^{*}$. Then $U \cap A=U^{*} \times A^{*}$ and $W \cap A=W^{*} \times A^{*}$. Since $U \cap A$ and $W$ are Baire spaces, $U^{*}, W^{*}$, and $A^{*}$ are Baire spaces. Since $\prod_{i \in F} X_{i}$ has property (P), $W \cap A$ is a Baire space. Since $A$ is a dense subset of $X$ with the property of Baire, this implies that $X \sim A$ is of the first category in $X$.

1.4 Proposituon. Suppose that, for each $i$ in $I$, the set $\left\{j \in I: X_{j}\right.$ is homeomorphic with $\left.X_{i}\right\}$ is infinite. Then 1.1.1 holds.

The proof of 1.2 establishes 1.4 , if we choose $U$ and $W$ so that $U^{*}$ is homeomorphic with $W^{*}$.

Combining 1.2, 3(4) and 3(9) of [4], and Theorem 3 of [2], we have the following generalization of Theorem 4 of [2].

1.5 THEOREM. Suppose each $X_{i}$ is either a Baire space with a countable pseudo-base or is weakly $\alpha$-favorable. Then $X$ is a Baire space and 1.1.1 holds. 
Remarks. (1) Proposition 1.4, when $I$ is countable, follows from the theorem in [3].

(2) If, in 1.2 , the $X_{i}$ are arbitrary topological spaces, then 1.1.1, with the phrase "has the property of Baire in $X$ " replaced by "is a Borel set in $X^{\prime}$, holds. For then $A^{*}$ is a dense Borel subset of $\prod_{i \in I \sim F} X_{i}$ such that $A^{*}$ is a Baire space. Hence $\left(\prod_{i \in I \sim F} X_{i}\right) \sim A^{*}$ is of the first category in $\prod_{i \in I \sim F} X_{i}$.

2. A topological space $(X, \mathscr{T})$ is called $\alpha$-favorable [1] if there is a function $\varphi: \mathscr{T}^{*} \rightarrow \mathscr{T}^{*}$, where $\mathscr{T}^{*}=\{U \in \mathscr{T}: U \neq \varnothing\}$, such that $\varphi(U) \subset U$ for all $U$ in $\mathscr{T}^{*}$ and, if $\gamma: N \rightarrow \mathscr{T}^{*}$ (here $N$ denotes the set of natural numbers) is such that $\gamma(n+1) \subset \varphi(\gamma(n))$ for all $n$ in $N$, then $\bigcap\{\gamma(n): n \in N\} \neq \varnothing$.

In [4], a related class of spaces, the class $\mathscr{C}_{w}$ of weakly $\alpha$-favorable spaces, is considered. It is shown that $\mathscr{C}_{w}$ has a number of reasonable properties (3(1) through $3(10))$. It would be of interest to know which of these properties the class $\mathscr{C}$ of $\alpha$-favorable spaces has (it is easily verified that $\mathscr{C}$ satisfies (1), (2), (4), (5), (7), (9), and (10)). In this section we prove that $\mathscr{C}$ satisfies (6) and half of the statement obtained when the terms " $\alpha$-favorable" and "weakly $\alpha$-favorable" are interchanged in (3).

2.1 Proposition. (a) If $(X, \mathscr{T})$ is a dense $G_{\delta}$ subset of a quasi-regular, $\alpha$-favorable space $(Y, \mathscr{U})$, then $X$ is $\alpha$-favorable.

(b) If $(X, \mathscr{T})$ is pseudo-complete [2], then $X$ is $\alpha$-favorable.

Proof. If $X$ satisfies the hypothesis of either (a) or (b), then $X$ satisfies the following condition.

2.2. There is a sequence $\left(\varphi_{n}\right)_{n \in N}$ such that, for each $n$ in $N, \varphi_{n}: \mathscr{T}^{*} \rightarrow$ $\mathscr{T}^{*}, \varphi_{n}(U) \subset U$ for all $U$ in $\mathscr{T}^{*}$, and if $\gamma: N \rightarrow \mathscr{T}^{*}$ is such that $\gamma(n+1) \subset$ $\varphi_{n}(\gamma(n))$ for all $n$ in $N$, then $\bigcap\{\gamma(n): n \in N\} \neq \varnothing$.

This is easily verified if $X$ is pseudo-complete. If $X$ satisfies the hypothesis of (a), then a simpler version of the argument used in proving 3(6) of [4] establishes 2.2. In detail: Let $\mathscr{R}$ denote the family of all regular elements of $\mathscr{U}$. Since $Y$ is quasi-regular, we may assume that $\varphi(U) \in \mathscr{R}$ for all $U$ in $\mathscr{U}^{*}$, where $\varphi$ is the function guaranteed by the definition of $\alpha$-favorable. Suppose $X=\bigcap\left\{G_{n}: n \in N\right\}$, where $G_{n} \in \mathscr{U}$ for all $n$ in $N$. Define $\gamma: \mathscr{T} \rightarrow \mathscr{U}$ so that $X \cap \gamma(U)=U$ for all $U$ in $\mathscr{T}$. Define $\varphi_{n}$ by letting $\varphi_{n}(U)=X \cap \varphi\left(\gamma(U) \cap G_{n}\right)$ for all $U$ in $\mathscr{T}^{*}$. Then $\left(\varphi_{n}\right)_{n \in N}$ satisfies 2.2 .

And, if 2.2 holds, then we may assume that $\varphi_{n+1}(U) \subset \varphi_{n}(U)$ for all $n$ in $N$ and all $U \in \mathscr{T}^{*}$; if necessary, replace $\left(\varphi_{n}\right)_{n \in N}$ by $\left(\psi_{n}\right)_{n \in N}$, where $\psi_{1}=\varphi_{1}$ and $\psi_{n+1}=\varphi_{n+1} \circ \psi_{n}$ for all $n$ in $N$.

We shall show that any space which satisfies 2.2 is $\alpha$-favorable. It is convenient to introduce the following condition.

2.3. There is a sequence $\left(\mathscr{P}_{n}\right)_{n \in N}$ of pseudo-bases for $X$ such that, if $P_{n} \in \mathscr{P}_{n}$ for all $n$ in $N$, then int $\left[\bigcap\left\{P_{n}: n \in N\right\}\right]=\varnothing$. 
Case 1. Suppose $X$ satisfies 2.3. We may assume that (1) $X \in \mathscr{P}_{1}$, and (2) for each $n$ in $N, \mathscr{P}_{n+1}$ refines $\mathscr{P}_{n}$. For $n$ in $N$, let

$$
\mathscr{B}_{n}=\left\{U \in \mathscr{T}^{*}: \text { There is } P_{n} \text { in } \mathscr{P}_{n} \text { such that } U \subset P_{n}\right\}
$$

and let $\mathscr{A}_{n}=\mathscr{B}_{n} \sim \mathscr{B}_{n+1}$. Since (2) holds, $\mathscr{A}_{i} \cap \mathscr{A}_{j}=\varnothing$ if $i \neq j$. Since (1) and 2.3 hold, $\mathscr{T}^{*}=\bigcup\left\{\mathscr{A}_{n}: n \in N\right\}$. For $U$ in $\mathscr{A}_{n}$, let $\varphi(U)$ be an element of $\mathscr{P}_{n+1}$ such that $\varphi(U) \subset \varphi_{n}(U)$. Suppose, now, that $\gamma: N \rightarrow \mathscr{T}^{*}$ is such that $\gamma(n+1) \subset \varphi(\gamma(n))$ for all $n$ in $N$. Then there is $j: N \rightarrow N$ such that $\gamma(n) \in$ $\mathscr{A}_{j(n)}$. And, since $\gamma(n+1) \subset \varphi(\gamma(n)) \in \mathscr{B}_{j(n)+1}, j(n)<j(n+1)$. Therefore $\gamma(n+1) \subset \varphi_{n}(\gamma(n))$ for all $n$ in $N$ and $\bigcap\{\gamma(n): n \in N\} \neq \varnothing$.

Case 2. Suppose that no $U$ in $\mathscr{T}^{*}$ satisfies 2.3. Suppose $U \in \mathscr{T}^{*}$ and define, by induction, a sequence $\left(\mathscr{U}_{n}\right)_{n \in N}$ such that, for each $n$ in $N$, $\mathscr{U}_{n} \subset\left\{V \in \mathscr{T}^{*}: V \subset U\right\}, \varphi_{n}\left[\mathscr{U}_{n}\right]$ is a disjoint family such that $\bigcup \varphi_{n}\left[\mathscr{U}_{n}\right]$ is dense in $U$, and $\mathscr{U}_{n+1}$ refines $\varphi_{n}\left[\mathscr{U}_{n}\right]$. Since $U$ does not satisfy 2.3 , there is a sequence $\left(U_{n}\right)_{n \in N}$ such that, for each $n$ in $N, U_{n} \in \mathscr{U}_{n}, U_{n+1} \subset \varphi_{n}\left(U_{n}\right)$, and $\operatorname{int}\left[\bigcap\left\{U_{n}: n \in N\right\}\right] \neq \varnothing$. Let $\varphi(U)=\operatorname{int}\left[\bigcap\left\{U_{n}: n \in N\right\}\right]$. Suppose $\gamma: N \rightarrow \mathscr{T}^{*}$ is such that $\gamma(n+1) \subset \varphi(\gamma(n))$ for all $n$ in $N$. For each $n$, there is $V_{n}$ in $\mathscr{T}^{*}$ such that $\varphi(\gamma(n)) \subset \varphi_{n}\left(V_{n}\right) \subset V_{n} \subset \gamma(n)$. So $V_{n+1} \subset \gamma(n+1) \subset$ $\varphi(\gamma(n)) \subset \varphi_{n}\left(V_{n}\right)$, and $\bigcap\{\gamma(n): n \in N\}=\bigcap\left\{V_{n}: n \in N\right\} \neq \varnothing$.

Case 3. Suppose neither Case 1 nor Case 2 holds. Then there are $U, V$ in $\mathscr{T}^{*}$ such that $U \cap V=\varnothing, U \cup V$ is dense in $X, U$ satisfies 2.3 and no nonempty open subset of $V$ satisfies 2.3. Therefore $U$ and $V$, and hence $X$, are $\alpha$-favorable.

2.4 Remarks. (1) It follows from 2.1 (a) that, if $X$ is a Baire space which is a dense subset of the quasi-regular, $\alpha$-favorable space $Y$, and $X$ has the property of Baire in $Y$, then $X$ is $\alpha$-favorable.

(2) A $G_{\delta}$ subset $X$ of an $\alpha$-favorable space $Y$ need not be $\alpha$-favorable even if $Y$ is metrizable, $X$ is a Baire space, and $X$ is a closed subset of $Y$. (Let $X$ be a subspace of the real line $R$ which is a Baire space that is not $\alpha$-favorable, and let $Y=R^{2} \sim\left\{(x, y) \in R^{2}: x \notin X, y=0\right\}$.)

\section{REFERENCES}

1. G. Choquet, Lectures on analysis. Vol. I: Integration and topological vector spaces, Benjamin, New York, 1969. MR 40 \#3252.

2. J. C. Oxtoby, Cartesian products of Baire spaces, Fund. Math. 49 (1960/61), 157166. MR 25 \#4055; 26, 1453.

3. M. Bhaskara Rao and K. P. S. Bhaskara Rao, A category analogue of HewittSavage zero-one law, Proc. Amer. Math. Soc. 44 (1974), 497-499.

4. H. E. White, Jr., Topological spaces that are $\alpha$-favorable for a player with perfect information, Proc. Amer. Math. Soc. (to appear).

251 N. Blackburn Road, Rt. \#5, Athens, Ohio 45701 